\title{
Interactive Digital Logic Laboratory for K-12 Students (Work in Progress)
}

\section{Dr. Rohit Dua, Missouri University of Science \& Technology}

ROHIT DUA, Ph.D is an Associate Teaching Professor in the Department of Electrical and Computer Engineering at the Missouri University of Science and Technology and Missouri State University's Cooperative Engineering Program. His research interests include engineering education. (http://web.mst.edu/ $/$ rdua/) 


\title{
Interactive Digital Logic Laboratory for K-12 Students (Work in Progress)
}

\begin{abstract}
The goal of this project is to address and contribute to the ever-growing demand to develop innovative and interactive education modules, catered towards K-12 students, which will encourage them to pursue a career path in computer engineering, a STEM field area, upon graduating from high school. Even though kids are experienced in using consumer electronic gadgets, they rarely understand the basics of how these devices were built. Exposing them to the foundations of computer hardware, may encourage them to think about how basic computer operations are performed. In addition to developing multiple tools, to teach kids about the working of logic gates, decimal-to-binary conversion, and representing positive and negative numbers, in computers, the project strives to make the tools interactive and fun to use.
\end{abstract}

\section{Introduction}

The Introduction to Computer Engineering course is a fundamental first course for computer and electrical engineering and computer science majors. The covered course material is used to learn about advanced computer engineering system design via subsequent computer engineering courses. Such systems are prevalent, in our day-to-day lives, in the form of consumer electronic products. Continuing trend in the industry and academia is to develop methods and techniques, which could encourage children to pursue engineering after graduating from high school [1]. Several programs exist that cater to this growing trend. One such program is Project Lead The Way (PLTW) [2]. Its engineering program, which is one of the three upper level programs, has been adopted by many schools, but is restricted to grades 9-12 [2]. An important question to answer is: "How do we show children, of all ages, elements of engineering so that they get excited about pursuing a career in engineering?". Also, the question is not about just demonstrating technology, but having children interact with devices that help them understand the basics ideas and engineering principles, which are used to build devices and technologies that they see all around them. That idea and thought led to the start of this project. While the concepts, covered in the introductory course, are challenging, even for a Sophomore level major, the question was: "Can we teach the same concepts to kids in a more fun and interactive way?". Therefore, it was decided to build devices, which would be interactive and visual, and easy for kids to comprehend. The Introduction to Computer Engineering course teaches students concepts ranging from basic blocks, which are used to build bigger blocks/systems, to complete simple system design. It was decided to incorporate the same methodology to teach kids about basics of computer engineering. Therefore, the initial designed tools would teach kids about the basic blocks. Subsequent implements would concentrate on bigger blocks. The next section concentrates on two devices, designed and constructed at Missouri University of Science and Technology, which have been field-tested. Feedback received is discussed in the following section leading to a brief discussion and conclusion.

\section{Developed Educational Tools}

From the onset of the project, it was decided that devices would be created and tested in progression as mentioned in the design methodology discussed above. Following is a description of the two completed devices, and their usage, which have been field-tested: 
- Basic Digital Logic Gates Emulator: A Learning Tool for Kids and Adults: The goal of this venture was to find a fun and innovative way to teach children about digital logic gates. An interactive system was designed, built and demonstrated that allows the user to understand the functioning of basic 2-input logic gates. The interactive system comprises of the facility to select, by the teacher, the 2-input gate under study and the method to excite the logic gate inputs. It was desired to make the learning tool fun with an innovative method, to change the gate input logic level, which would mimic a "magic show". Figure 1 shows the typical usage of the device. As seen in Figure 1, top-left image, the light falling on two photo-resistors is translated, using an electronic circuit, into logic 0 , which is then applied to the gate-under-test housed in a separate digital circuit unit. Blocking the light, by using hands, one can apply logic 1 to either one or both, the gate input/s. Depending on the gate-under-test, the LED matrix will light up for one of the four possible input combinations. The system is restricted to only 2-input gates. The teacher can choose the gate-under-test. A student must try to figure out what type of logic gate is under-test by trying different combinations of possible inputs applied by blocking the light with their hands.

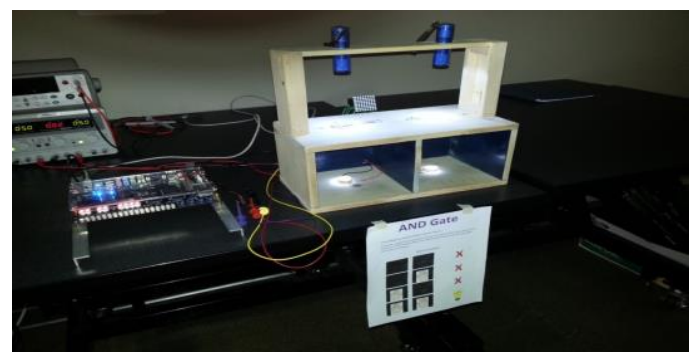

AND gate with no input

(0 hands) LED matrix is off

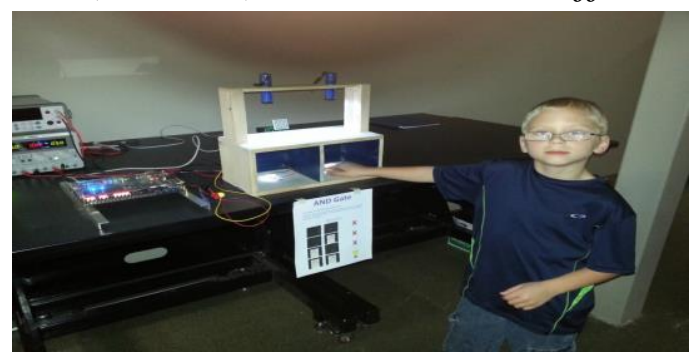

AND gate with one input (Right hand) LED matrix is off

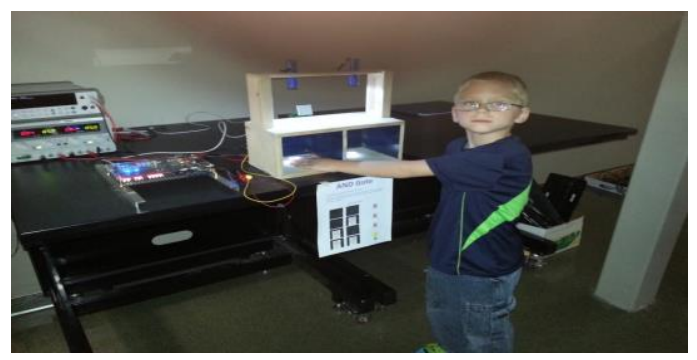

AND gate with one input (Left hand) LED matrix is off

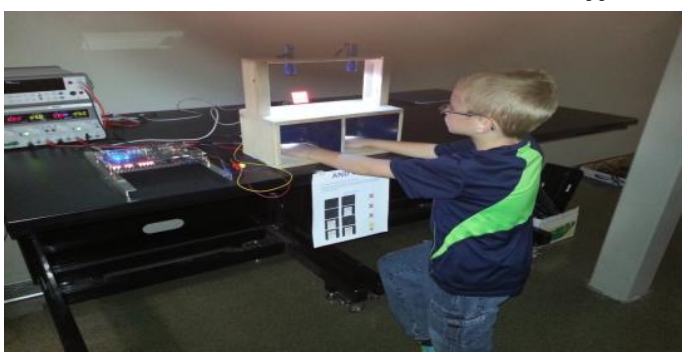

AND gate with two inputs (both hands) LED matrix is on

Figure 1: Typical usage of the logic gates emulator for a two-input AND gate

As the student tries different gates, the expectation is that the student will gain insight into how the basic logic gates (AND, OR, NOR, NAND, XOR, XNOR) vary in their functionality. An understanding of fundamental gates is crucial foundational step in understanding how more complex digital systems are created from simple logic gates. Figure 2 shows the informational slides developed to help aid in the user's understanding 
of the system usage. With multiple tries, it is expected that a student can operate the testing and learning, of a logic gate behavior, independently or with minimal help from the teacher.

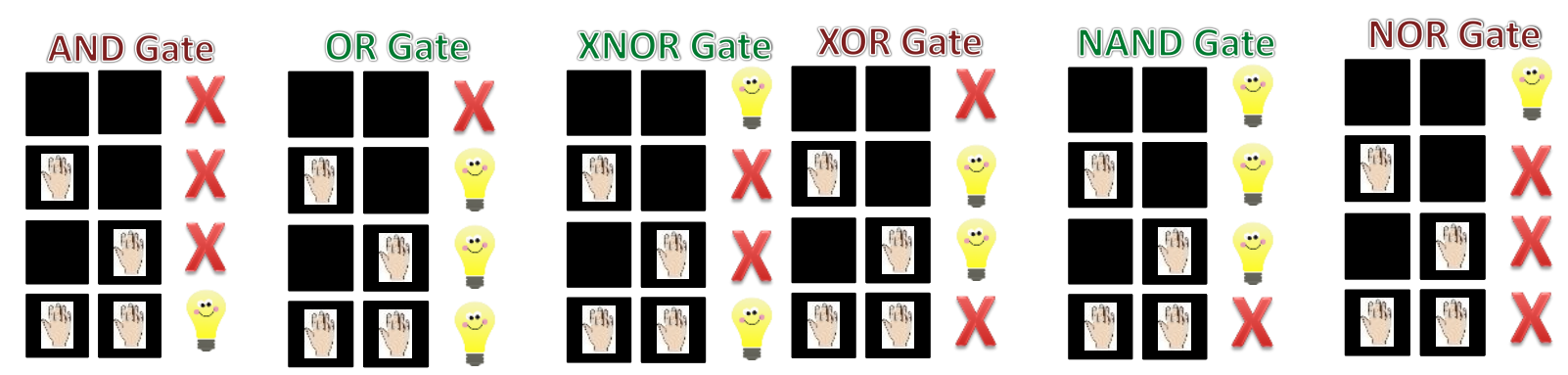

Figure 2: Informational slides developed to aid in learning of how basic logic gates work. These slides are supposed to be used in conjunction with the system as seen in Figure 1.

- Binary-to-Decimal Conversion Emulator: The goal of this project goal was to develop a fun way to teach children about signed and unsigned decimal-to-binary number conversion. The hardware constructed 8-bit binary-to-decimal conversion system allows a user to test an 8-bit unsigned (positive) and signed (positive/negative) numbers. It is important for students to understand how positive numbers are represented in binary. When factoring in negative numbers, the same of group of numbers (8-bits) must allow for both positive and negative numbers to be represented. The system can toggle between unsigned number and signed number domain using a switch. Before students can attempt testing binary numbers, a short verbal tutorial, by an instructor, explains the use of decimal weights, as seen in Figure 3, for each binary bit position, which are used to convert a decimal number into binary.

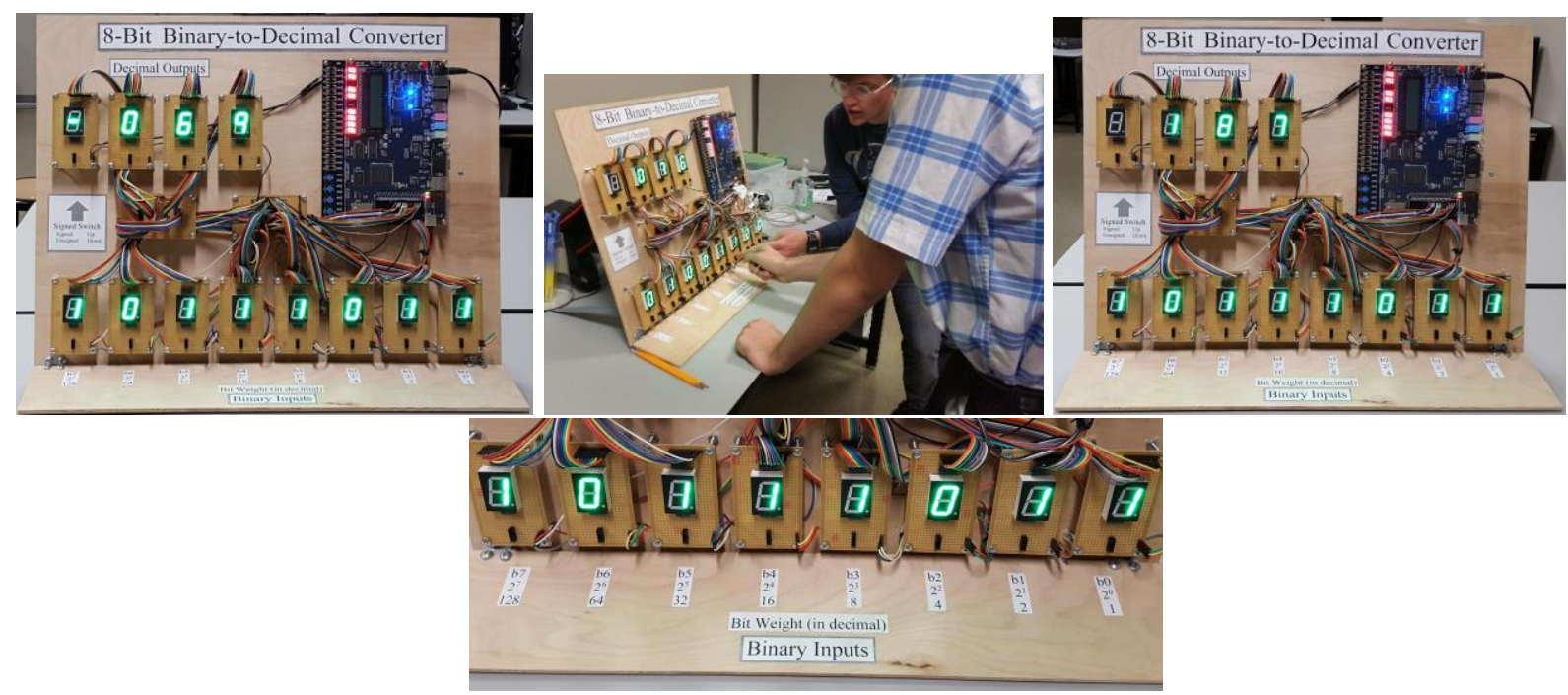

Figure 3: The Decimal-To-Binary Emulator. The upper images shows how a particular 8-bit binary combination can be considered as a positive number or a negative number. The lower image shows the switches, which can be used to either apply ' 1 ' or ' 0 ' for a particular bit position. Note the emphasis of binary bit weights, which are crucial to covert a decimal number into binary. The top middle image depicts a typical interaction with the emulator 
Students first try to convert a positive number into binary, toggling the appropriate switches to place a bit 1 in respective bit-location. As the binary number is created, its decimal equivalent is displayed, which provide visual confirmation of the decimal equivalent. Figure 3 also shows students interacting with the emulator. After gaining expertise in converting positive numbers to binary, a student can attempt converting that binary number into a negative number using the 2 s complement method. Students are shown the quick-way to convert the positive binary number into the corresponding negative number using the $2 \mathrm{~s}$ complement technique. As students learn the conversion technique, they can enter the negative number representation, into the system, to verify the decimal equivalent, thus getting instant feedback, which instills confidence in the technique usage and understanding of the negative number binary representation. Students are encouraged to explore the decimal number range, for an 8-bit binary number, for both unsigned and signed number domains.

\section{Outreach and Feedback:}

Since the planned laboratory is still in its infancy, the designed emulators have been field tested in informal settings. Below are some survey results for the two emulators, which were demonstrated at two different events:

- Logic Gates Emulator: The device was demonstrated at a Discover Engineering Day event. Users ranging from kids-to-adults used the device to understand the basic functioning of logic gates. Twenty children interacted with the device and were requested to fill out a survey at the end of their interactive sessions. The short survey consisted of four statements. Children were asked to rank how well they agreed (score of 10) or disagreed (score of 1) with each statement. Figure 4 shows the survey results, which imply a largely positive feedback. The $\mathrm{x}$ axis represents the score (1-10) and the y-axis represents the number of participants, for each score, for each statement asked.

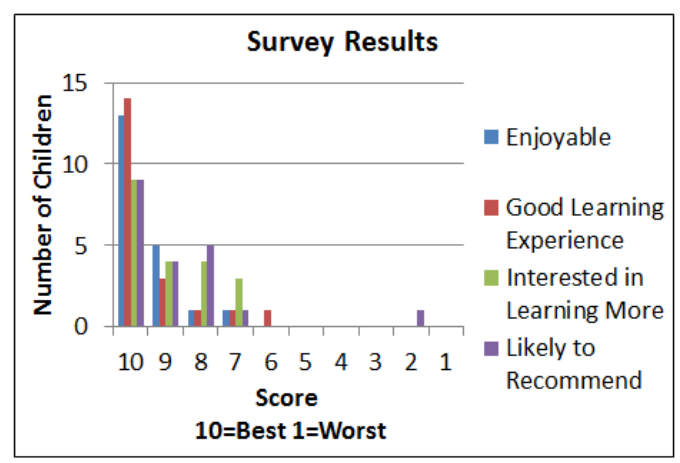

Figure 4: Logic gates emulator interaction survey results. Twenty Participants were asked to respond to four statements as mentioned above for this survey.

Most children found it an enjoyable and a good learning experience. They were, also, more likely to recommend the session to others. Many showed interest in learning more.

- Binary-To-Decimal Conversion Emulator: In addition to the device being demonstrated at a number of events, in an informal setting, such as the Maker-Faire and Discover Engineering Day, the emulator was demonstrated at an Electrical Engineering Laboratory event conducted for high school students in-order to expose them to the field of Electrical Engineering. Thirty-two 
students participated in the lab event. As part of the exit survey conducted, students were asked their views on three statements to assess the impact the B-to-D converter made on their understanding of the underlying concept. Figure 5 depicts the results of the survey, which clearly show that students found the emulator to be a great platform for learning about how positive and negative decimal integers are represented in binary.
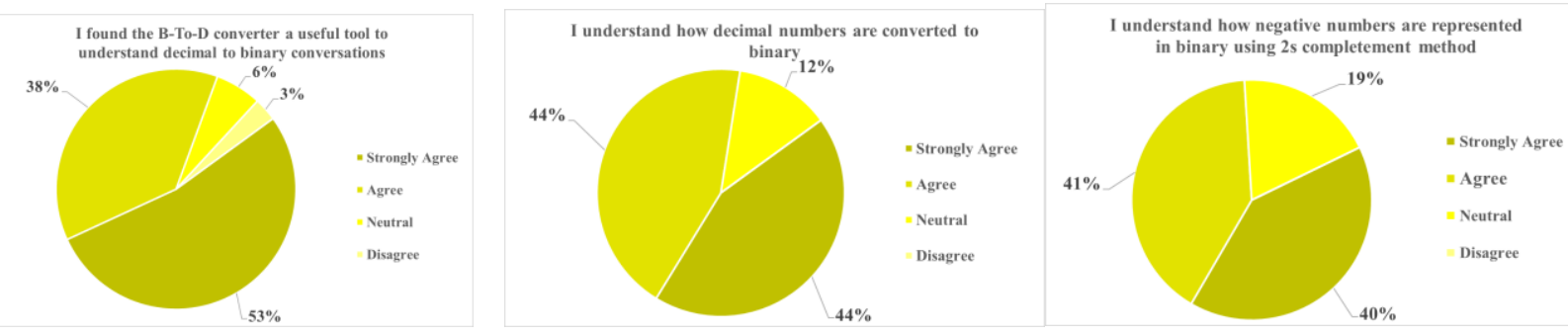

Figure 5: B-to-D emulator interaction survey results. Thirty two participants were asked to respond to three statements as mentioned above for this survey.

Within a few minutes, students could convert decimal numbers into binary, a task that usually takes a lecture period in a standard introduction to computer engineering course.

\section{Continuing Work and Conclusion}

The development, construction and field-testing of two basic educational units has had a resounding success in teaching basics of computer engineering to K-12 students. This accomplishment has laid a strong foundation to the planned interactive laboratory. As more devices are constructed and field-tested, the successful development, and continued implementation, of the laboratory will be a priority. One crucial goal is to incorporate learning components, via interactive educational tools, form every major learning unit found in an introductory computer-engineering course. Even though, currently, the devices are field tested in major events, in town, and through in-lab classes, the ultimate goal is to take these tools to schools and hold laboratory classes. An important additional tool design requirement was its portability, so that the tools can easily be transported to venues for planned workshops, at schools, and other events, such as, the Maker-Faire. The designed tools cater to a wide variety of audience ranging from elementary level students, to a more mature group of kids, and even adults who are interested in learning more about basics of computer engineering. Moreover, tools are designed and built by undergraduate electrical engineering students, who gain skill, via such experiential learning based projects.

\section{References}

1. U.S. Department of Education Webpage, "Science, Technology, Engineering and Math: Education for Global Leadership," (accessed 7 February 2017). Available https://www.ed.gov/Stem

2. Project Lead The Way, "PLTW Engineering (9-12) Webpage," (accessed 7 February 2017). Available https://www.pltw.org/our-programs/pltw-engineering 